\title{
Expanding the endoscopic transpterygoid corridor to the petroclival region: anatomical study and volumetric comparative analysis
}

\author{
Jacob L. Freeman, MD, ${ }^{1}$ Raghuram Sampath, MD, ${ }^{1}$ Steven Craig Quattlebaum, MD, ${ }^{2}$ \\ Michael A. Casey, DO, ${ }^{1}$ Zach A. Folzenlogen, MD, ${ }^{1}$ Vijay R. Ramakrishnan, MD, ${ }^{2}$ and \\ A. Samy Youssef, MD, $\mathrm{PhD}^{1,2}$ \\ Departments of ${ }^{1}$ Neurosurgery and ${ }^{2}$ Otolaryngology, University of Colorado, Aurora, Colorado
}

OBJECTIVE The endoscopic endonasal transmaxillary transpterygoid (TMTP) approach has been the gateway for lateral skull base exposure. Removal of the cartilaginous eustachian tube (ET) and lateral mobilization of the internal carotid artery (ICA) are technically demanding adjunctive steps that are used to access the petroclival region. The gained expansion of the deep working corridor provided by these maneuvers has yet to be quantified.

METHODS The TMTP approach with cartilaginous ET removal and ICA mobilization was performed in 5 adult cadaveric heads (10 sides). Accessible portions of the petrous apex were drilled during the following 3 stages: 1) before ET removal, 2) after ET removal but before ICA mobilization, and 3) after ET removal and ICA repositioning. Resection volumes were calculated using 3D reconstructions generated from thin-slice CT scans obtained before and after each step of the dissection.

RESULTS The average petrous temporal bone resection volumes at each stage were $0.21 \mathrm{~cm}^{3}, 0.71 \mathrm{~cm}^{3}$, and $1.32 \mathrm{~cm}^{3}$ ( $p<0.05$, paired t-test). Without ET removal, inferior and superior access to the petrous apex was limited. Furthermore, without ICA mobilization, drilling was confined to the inferior two-thirds of the petrous apex. After mobilization, the resection was extended superiorly through the upper extent of the petrous apex.

CONCLUSIONS The transpterygoid corridor to the petroclival region is maximally expanded by the resection of the cartilaginous ET and mobilization of the paraclival ICA. These added maneuvers expanded the deep window almost 6 times and provided more lateral access to the petroclival region with a maximum volume of $1.5 \mathrm{~cm}^{3}$. This may result in the ability to resect small-to-moderate sized intradural petroclival lesions up to that volume. Larger lesions may better be approached through an open transcranial approach.

https://thejns.org/doi/abs/10.3171/2017.1.JNS161788

KEY WORDS endoscopic; endonasal; transpterygoid approach; carotid mobilization; petrous temporal bone; anatomy

$\mathrm{O}$ VER the last decade, endonasal endoscopic approaches to the petrous apex have been thoroughly evaluated in cadavers and are now being used in the operating room for select diseases involving the lateral skull base. ${ }^{3,9,10,14,26,28}$ The roadmap for the ventral skull base varies greatly from that encountered via traditional transcranial routes to the petrous apex, offering a different set of obstacles to overcome. Approaches to the lateral skull base are generally divided into 2 main modules: 1 ) infrapetrous to the petrous apex and petroclival region, and 2) suprapetrous to the cavernous sinus and infratemporal fossa. ${ }^{14}$ In the absence of pathology, lateral mobilization of the paraclival ICA and anterior genu is paramount for superior access to the petrous apex. ${ }^{28}$ Partial removal of the eustachian tube (ET) may be required to adequately expose and mobilize the internal carotid artery (ICA) and access the medial petroclival region. ${ }^{2}$ These maneuvers are technically demanding and pose the risk of vascular

ABBREVIATIONS ET = eustachian tube; IAC = internal acoustic canal; ICA = internal carotid artery; PPF = pterygopalatine fossa; PPG = pterygopalatine ganglion; TMTP $=$ transmaxillary transpterygoid.

SUBMITTED July 6, 2016. ACCEPTED January 19, 2017.

INCLUDE WHEN CITING Published online July 21, 2017; DOI: 10.3171/2017.1.JNS161788. 

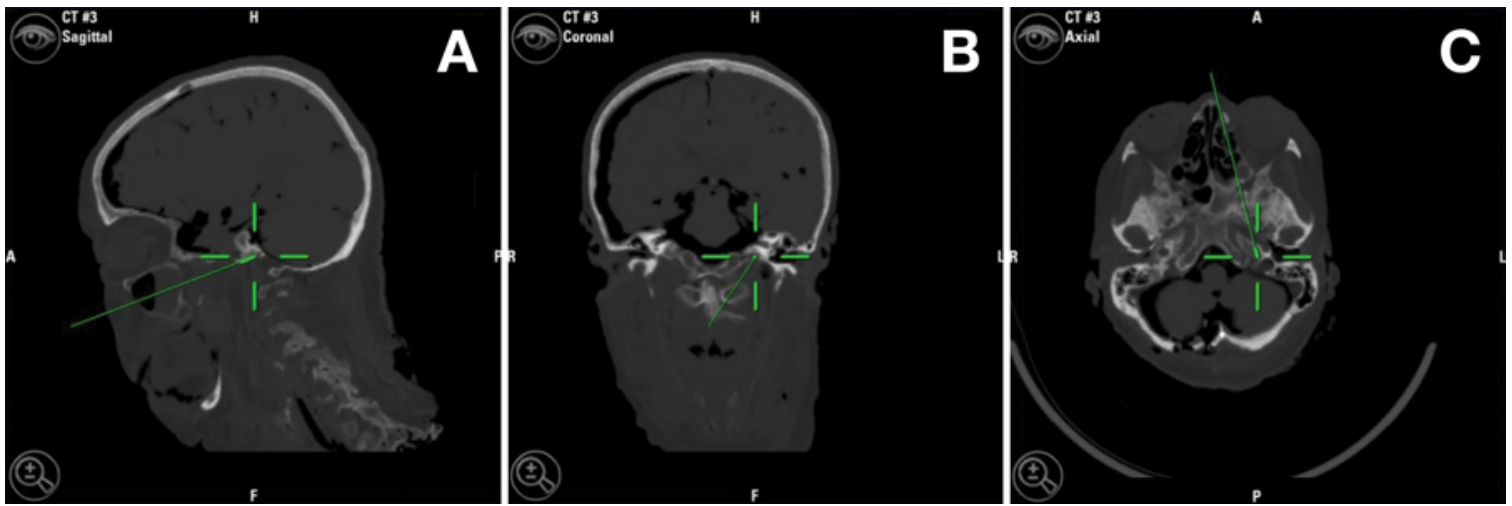

FIG. 1. Brainlab neuronavigation was used during the cadaveric dissections to assist in the standardization of dissection boundaries. As an example, the probe pointing to the lower extent of exposure (jugular foramen) is shown in the sagittal (A), coronal (B), and axial (C) planes.

injury to the ICA or altered hearing associated with partial removal of the ET. Despite the adoption and performance of these surgical steps by expert surgeons, the blueprint is still missing morphometric data about the added exposure provided by mobilizing such critical structures. When performing the ventral approach to the lateral skull base, the question arises whether to circumnavigate these structures in their normal anatomical positions, mobilize (in the case of the ICA), remove (in the case of the ET), or perform some combination of these maneuvers to improve access. The choice depends on the specific type and location of the pathology, coupled with the known risks associated with each approach. ${ }^{13}$ We sought to quantify the difference in maximal petrous apex resection volumes and identify resection limits using the standard transmaxillary transpterygoid (TMTP) approach before and after cartilaginous ET resection and before and after ICA mobilization in cadavers. Our aim was to identify specific areas of the petrous apex that were accessed at each stage and assess in a volumetric fashion the window created to the petroclival region through an endoscopic endonasal approach.

\section{Methods}

Five latex-injected adult cadaveric heads (10 petrous apices) were initially dissected for this anatomical study. A sixth head was dissected with an emphasis on demonstrating the extra- and intradural landmarks of the exposure. Endoscopic dissections were performed using a Karl Storz endoscope that was $4 \mathrm{~mm}$ in diameter and $18 \mathrm{~cm}$ in length, with $0^{\circ}, 30^{\circ}$, and $45^{\circ}$ lenses. All dissections were performed under navigation guidance (Fig. 1).

\section{TMTP Approach}

We performed the TMTP approach bilaterally as follows. We began our dissection with bilateral inferior and middle turbinectomies, maxillary antrostomy, total ethmoidectomy, and sphenoidotomy for wide exposure. A $1.5-\mathrm{cm}$ posterior septectomy was performed to improve angles of access from the contralateral nostril, decrease contamination of the endoscope along the posterior septum, and allow for ease of a bimanual, 2-sur- geon approach. The bone of the sphenoid rostrum was removed, ${ }^{5}$ creating a wide common sphenoidotomy. The posteromedial wall of the maxillary sinus was removed, exposing the pterygopalatine fossa (PPF). ${ }^{17}$ The sphenopalatine artery and vidian nerve were transected to allow lateral retraction of the PPF contents, thereby exposing the pterygoid base and plates. The medial pterygoid plate was drilled flush with the foramen rotundum, and the lateral pterygoid plate was drilled flush with the foramen ovale as described by Kassam et al. ${ }^{14}$ The lateral wall of the sphenoid sinus was removed to expose the dura of the medial wall of the cavernous sinus to the level of the V2 and V3 segments of the trigeminal nerve as they exit their respective foramina. The vidian canal was then identified and followed with a diamond drill posteriorly to locate the lacerum segment of the ICA. ${ }^{15}$ Bone overlying the lacerum and distal horizontal petrous segment of the ICA was thinned with a diamond bur and removed using rongeurs. To complete the necessary ICA exposure, the paraclival and cavernous carotid segments were then carefully unroofed with the aid of Kerrison rongeurs. To maximize carotid mobilization and petrous apex exposure and resection, the petrolingual ligament was released, ${ }^{2,30}$ the pharyngobasilar fascia was freed from the anterior genu, ${ }^{21}$ and the fibrocartilaginous tissue along the inferior portion of the foramen lacerum was removed (Figs. 2A and 3A). ${ }^{28} \mathrm{~A}$ high-speed electric drill (Stryker Sable drill for the first 5 dissections and Primado 2 Drill [NSK America Corp.] for the remaining dissection) with a 3-mm round diamond bur was then angled posterolaterally behind the paraclival ICA to drill the maximum accessible portion of the anteromedial petrous apex. Attempts at drilling inferior to the genu and distal horizontal petrous segment of the ICA (the infrapetrous corridor) were limited by the cartilaginous ET (Fig. 2B). This was performed on the right side, and, given the very limited exposure, it was used as a reference for both sides. The first set of thin-cut CT scans was then obtained to be used as a template for baseline exposure.

\section{ET Resection}

The nasopharyngeal, pterygoid, and lacerum segments 
of the cartilaginous $\mathrm{ET}^{21}$ were resected, sparing the torus tubarius and thus opening the infrapetrous corridor to the petrous apex (Figs. 2C and $3 \mathrm{~A}$ and B). Leaving the ICA in place, the inferior petrous apex was entered by drilling in a superoposterior direction beneath the anterior genu, lacerum segment, and distal horizontal petrous ICA (Fig. 2C). A second set of thin-slice CT scans was obtained.

\section{ICA Mobilization}

Finally, the ICA was repositioned to further reveal the petrous apex. The paraclival, lacerum, and distal horizontal petrous segments of the ICA were retracted laterally, exposing the petrous apex (Figs. 2D and 3C). Brainlab neuronavigation was used to guide petrous apex removal. Resection boundaries were standardized to the foramen rotundum anterolaterally, the dura of the internal acoustic canal (IAC) posterolaterally, the top of the petrous temporal bone superiorly, the posterior fossa dura medially, and the top of the jugular foramen posteriorly (Fig. 1). Extra- and intradural exposures are shown in Figs. 2-4. The intradural exposure provided access to the petroclival region from the basilar artery medially to the IAC laterally, inferiorly to the jugular foramen and lower cranial nerves, and superiorly to the top of the pons and trigeminal nerve. A final set of thin-slice noncontrast CT scans of each head was obtained.

\section{Volumetric Analysis}

The thin-cut CT scans from each stage of the dissection were loaded into the Brainlab neuronavigation-planning unit to calculate resection volumes. The baseline head CT study obtained prior to drilling was first fused to the head CT scan obtained after drilling but before ET resection and before ICA mobilization. To calculate only the petrous apex resection volume, the postresection CT scan was overlaid onto the axial CT scan obtained prior to drilling, revealing the area of petrous apex that was resected. Next, the removed area (in $\mathrm{cm}^{2}$ ) of petrous apex in each axial slice was manually outlined, and the software was used to combine each slice together to formulate a resection volume $\left(\mathrm{in}^{3}\right)$. This process was repeated for each stage of the resection to calculate volumes of resection. A representative 3D model, generated from the axial slices of each CT scan obtained throughout the dissection, demonstrates the different areas of the petrous apex that were accessed at each stage of the resection (Fig. 5).

\section{Statistical Analysis}

One-tailed, paired t-tests were used to compare resection volumes from each sequential stage of dissection, and 2-tailed paired t-tests were used to compare left- versus right-side dissections at each stage, with an alpha at 0.05 to determine statistical significance.

\section{Results}

Tables 1 and 2 list the petrous apex resection volumes at each stage of the dissection and are represented graphically in Fig. 6. Before ET resection or ICA mobilization,
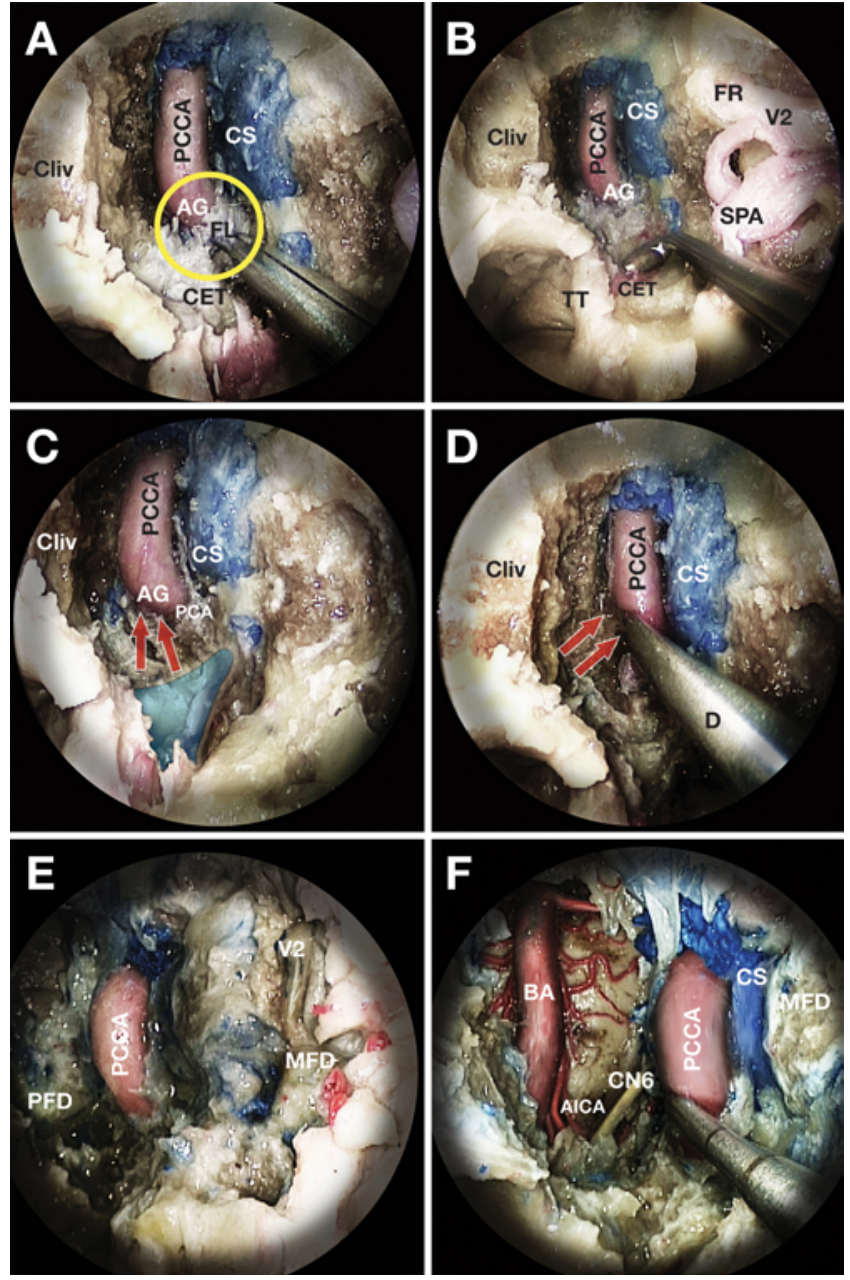

FIG. 2. Left TMTP approach. A: Pre-ET resection and pre-ICA mobilization. The left lateral clivus has been drilled to allow better, albeit minimal, access to the anterior-most portion of the petrous apex. Endoscopic scissors are used to release the fibrocartilaginous attachments of the lacerum segment of the ICA as well as the pharyngobasilar fascia attachments to the anterior genu to prepare for mobilization. The yellow circle indicates the area of focus. B: Curette pointing at superior portion of the cartilaginous ET. C: Post-ET resection and pre-ICA mobilization. The transpterygoid approach has been performed, and the cartilaginous ET has been removed, creating a large space (blue shading) for infrapetrous access to the lower petrous apex as indicated by the red arrows. D: Post-ET resection and post-ICA transposition with dissector (static vascular retractor can also be used as shown in Fig. 3), revealing more of the upper half of the petrous apex, which has been drilled in part (red arrows). E: Dural margins of the posterior and middle fossae are demonstrated after removal of ET and drilling of lower petrous apex. F: Minimal lateral retraction of the anterior genu after removal of the posterior fossa dura demonstrating the left abducens nerve in the prepontine cistern. $A G=$ anterior genu of the ICA; $A I C A=$ anterior inferior cerebellar artery; $\mathrm{BA}=$ basilar artery; $\mathrm{CET}=$ cartilaginous eustachian tube; Cliv = clivus; $\mathrm{CN6}=$ cranial nerve Vl; $\mathrm{CS}=$ medial cavernous sinus dura; $\mathrm{D}=$ dissector; $\mathrm{FL}$ = foramen lacerum; $F \mathrm{R}$ = foramen rotundum; $M F D=$ middle fossa dura; $P C A=$ distal horizontal petrous carotid artery; PCCA = paraclival carotid artery; $P F D=$ posterior fossa dura; SPA = sphenopalatine artery; TT = torus tubarius; V2 = maxillary nerve. 

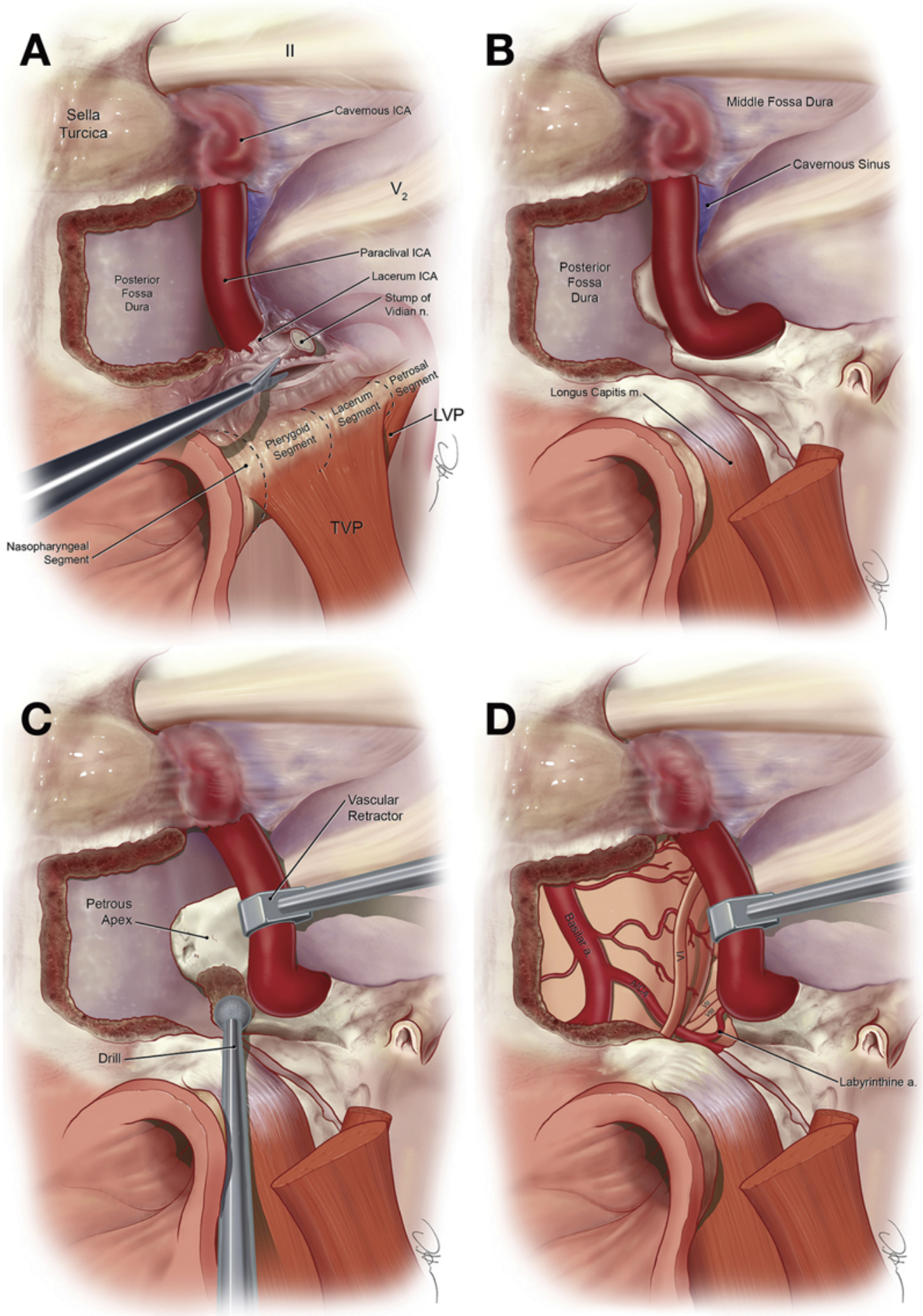

FIG. 3. A step-by-step illustration of the key steps of the approach with emphasis on ET resection and ICA mobilization for exposure of the petroclival region. A: Sharp transection of the fibrocartilaginous attachments of the lacerum segment of the ICA and the pharyngobasilar fascia attachments to the anterior genu is performed, thus untethering in preparation for transposition. B: Removal of the nasopharyngeal, pterygoid, and lacerum cartilaginous ET segments. The tensor veli palatini (TVP) and levator veli palatini (LVP) attachments are removed along with the ET. The torus tubarius is spared, as removal does not improve ipsilateral approach or access to the petrous apex. If a contralateral approach is desired, the torus tubarius can be removed, or the drill can be used to easily displace it inferiorly. C: Lateral mobilization of the paraclival ICA using a static vascular retractor revealing more of the upper petrous apex. The endonasal high-speed drill using a round diamond bur is used for petrous apex removal. D: The exposed petrous apex and posterior fossa dura have been removed, revealing the intradural exposure. a. = artery; AICA = anterior inferior cerebellar artery; $\mathrm{m}$. = muscle; $\mathrm{n} .=$ nerve; $\mathrm{II}=$ optic nerve; $\mathrm{VI}=$ abducens nerve; $\mathrm{VII}=$ facial nerve; $\mathrm{VIII}=$ vestibulocochlear nerve; $V_{2}=$ second branch (maxillary division) of the trigeminal nerve. Artist: David Aten. Published with permission from $A$. Samy Youssef, MD, PhD. 

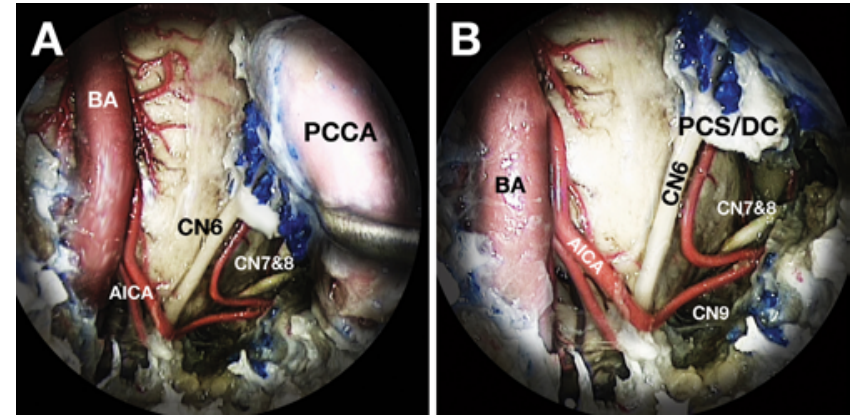

FIG. 4. Magnified intradural view of the left petroclival region after maximal lateral ICA retraction and dural resection using $0^{\circ}(\mathrm{A})$ and $30^{\circ}$ (B) endoscopes. Medially, the basilar artery (BA) is exposed, posterolaterally the CN VII/VIII complex (CN7\&8) can be seen within the IAC, superiorly the top of the pons is exposed, and inferiorly the top of $\mathrm{CN}$ IX (CN9) is seen exiting the brainstem. PCS/DC = posterior cavernous sinus/Dorello's canal.

the petrous apex resection volume was assessed on one side (right) per head and ranged from 0.18 to $0.25 \mathrm{~cm}^{3}$ (mean $0.21 \mathrm{~cm}^{3}$ ). After ET resection but before ICA mobilization, the resection volume ranged from $0.33 \mathrm{~cm}^{3}$ to $1.23 \mathrm{~cm}^{3}$ (mean $0.81 \mathrm{~cm}^{3}$ ) on the right and $0.37 \mathrm{~cm}^{3}$ to $0.80 \mathrm{~cm}^{3}$ (mean $0.61 \mathrm{~cm}^{3}$ ) on the left. The mean resection volume of both sides was $0.71 \mathrm{~cm}^{3}$. After static retraction of the ICA, resection volume ranged from 1.00 to $1.95 \mathrm{~cm}^{3}$ with a mean of $1.53 \mathrm{~cm}^{3}$ on the right and 0.91 to $1.45 \mathrm{~cm}^{3}$ with a mean of $1.12 \mathrm{~cm}^{3}$ on the left. The mean resection volume of both sides was $1.32 \mathrm{~cm}^{3}$. Assessing the difference between each stage revealed a mean additional petrous resection volume of $0.50 \mathrm{~cm}^{3}$ gained after ET resection but before ICA mobilization ( $\mathrm{p}=0.002)$, and an additional $0.61 \mathrm{~cm}^{3}$ resection volume after ICA mobilization $(\mathrm{p}<0.001)$.

Comparing right- and left-sided resection volumes after ET resection $(\mathrm{p}=0.266)$ and after ICA mobilization ( $\mathrm{p}$ $=0.780$ ), volume differences were not statistically different at $p>0.05$.

\section{Discussion}

Expanded endoscopic endonasal approaches to the petroclival region may require removal of the cartilaginous ET and mobilization of the ICA. In the current cadaveric study, each step allowed more bone resection and expanded the window to the petroclival region. Drilling is limited to the inferomedial portions of the petrous apex without carotid repositioning; however, when coupled with the maximum ICA lateralization, the entire petrous apex can be exposed and removed (Fig. 7). The intradural exposure provided access to the petroclival region from the basilar artery medially to the IAC laterally, inferiorly to the jugular foramen and lower cranial nerves, and superiorly to the top of the pons and trigeminal nerve.

\section{Approaches to the Petrous Apex and Petroclival Region}

Transcranial approaches to the lateral skull base have evolved to become the most direct access to the petrous apex and petroclival region. As skull base surgeons have
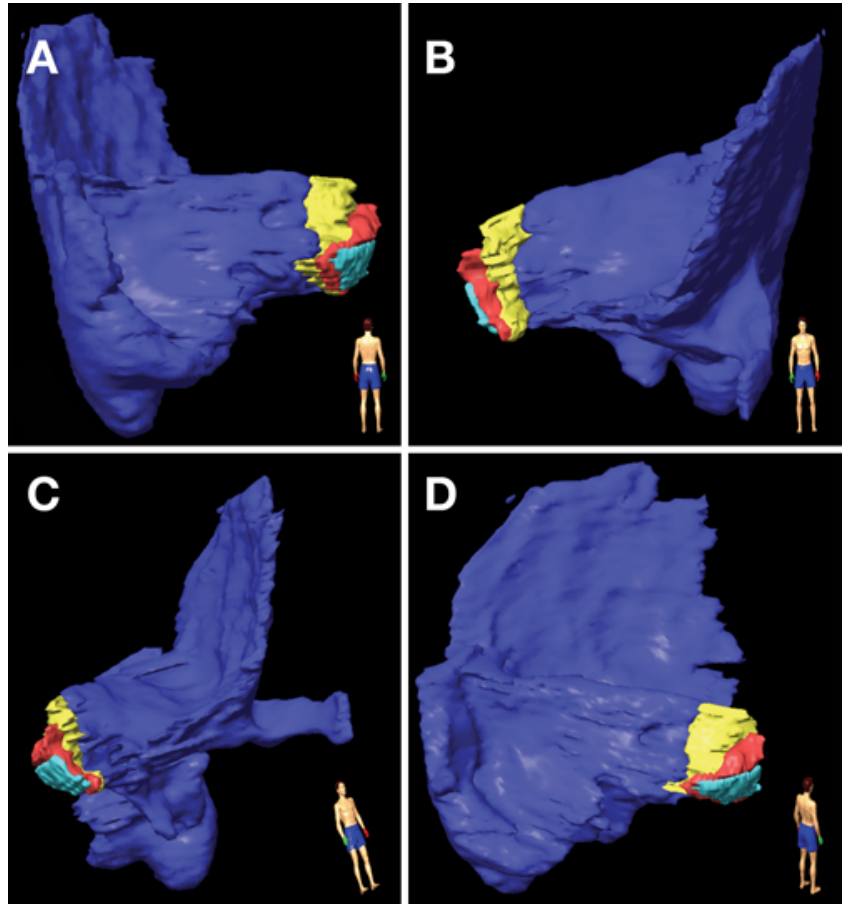

FIG. 5. 3D reconstructions of the left temporal bone from one of the cadaver dissections in which the TMTP approach was performed. The petrous apex was drilled before and after ET resection and paraclival ICA mobilization. Light blue represents the volume of petrous apex drilled via a medial transsphenoidal approach with a small amount of lateral clivus removed but before ET resection or ICA mobilization. Red demonstrates the amount of the petrous apex accessed after ET resection. Yellow demonstrates additional apiceal volume drilled after ET resection and carotid mobilization. Different views include posterior (A), anterior (B), anterior oblique (C), and posterior oblique (D) with a representative body figure in the bottom right corner of each quadrant to demonstrate head positioning.

gained more comfort and familiarity with these techniques, the utilization of expanded endonasal approaches has been extended to treat lesions involving the petroclival region. Given the complexity of regional anatomy and advanced technical maneuvers required, endoscopic surgery might be associated with high morbidity in less experienced hands. To optimize surgical planning, Kassam et al. ${ }^{14}$ and others ${ }^{10,11}$ have used classification systems based on the petrous and paraclival segments of the ICA for approaches to lesions in and around the petrous tem-

TABLE 1. Volume of petrous temporal bone resection before ET resection and before ICA mobilization (5 sides)

\begin{tabular}{cc}
\hline Specimen No. & Resection Vol $\left(\mathrm{cm}^{3}\right)$ \\
\hline 1 & 0.180 \\
\hline 2 & 0.201 \\
\hline 3 & 0.246 \\
\hline 4 & 0.241 \\
\hline 5 & 0.192 \\
\hline Mean & 0.213 \\
\hline
\end{tabular}


TABLE 2. Volume of petrous temporal bone resection after ET resection (10 sides)

\begin{tabular}{ccc}
\hline & \multicolumn{2}{c}{ Resection Vol $\left(\mathrm{cm}^{3}\right)$} \\
\cline { 2 - 3 } Specimen No. & Right & Left \\
\hline Before ICA mobilization & & \\
\hline 1 & 1.231 & 0.802 \\
\hline 2 & 0.334 & 0.374 \\
\hline 3 & 0.637 & 0.520 \\
\hline 4 & 0.930 & 0.696 \\
\hline 5 & 0.919 & 0.649 \\
\hline Mean & 0.810 & 0.608 \\
\hline 1 & & \\
\hline 2 & 1.947 & 1.330 \\
\hline 3 & 1.001 & 0.916 \\
\hline 4 & 1.481 & 0.912 \\
\hline 5 & 1.388 & 1.453 \\
\hline Mean & 1.810 & 0.981 \\
\hline
\end{tabular}

poral bone. Specific approaches to the petrous apex, without ICA mobilization include: 1) the medial transsphenoidal approach, ${ }^{5,14}$ reserved for cystic lesions that expand anteromedially into the sphenoid sinus; 2) infrapetrous approach, ${ }^{14}$ which involves drilling below the horizontal petrous ICA; 3) the trans-Meckel's cave route, ${ }^{10}$ which is accessed through the quadrangular space superior to the petrous ICA and lateral to the paraclival ICA; and 4) the translacerum approach, ${ }^{26}$ which involves removal of the fibrocartilaginous portions of the lacerum segment of the ICA to create a window into the inferior petrous apex lateral to the anterior genu and superior to the ET. Finally, the TMTP approach ${ }^{1,23,28}$ has been described for resection of petrous apex pathology. Although this approach can also be used without carotid mobilization to access portions of the petrous apex, transposition of the ICA provides maximal exposure of the petrous apex and petroclival region.

Several neurovascular structures have to be mobilized to open the surgical corridor to the petroclival region. To navigate the TMTP route, the sphenopalatine artery must be ligated and transected to facilitate lateral mobilization of the internal maxillary artery and other contents of the pterygopalatine fossa. ${ }^{2}$ The vidian nerve, which courses through the pterygoid canal on its way to the pterygopalatine ganglion (PPG) in the posterior PPF must also be sacrificed to release the PPG for lateral repositioning in order to expose the underlying pterygoid base and plates, which are then removed. At this point, one can reach small portions of the petrous temporal bone; however, access to most of this area remains obstructed by the paraclival and distal horizontal petrous segments of the ICA and the cartilaginous ET.

Therefore, the nasopharyngeal, pterygoid, and lacerum segments of the cartilaginous ET are resected to allow drilling of the temporal bone inferior to the lacerum and horizontal petrous carotid segments and to create extra space wherein the handle of the drill can be lowered. This

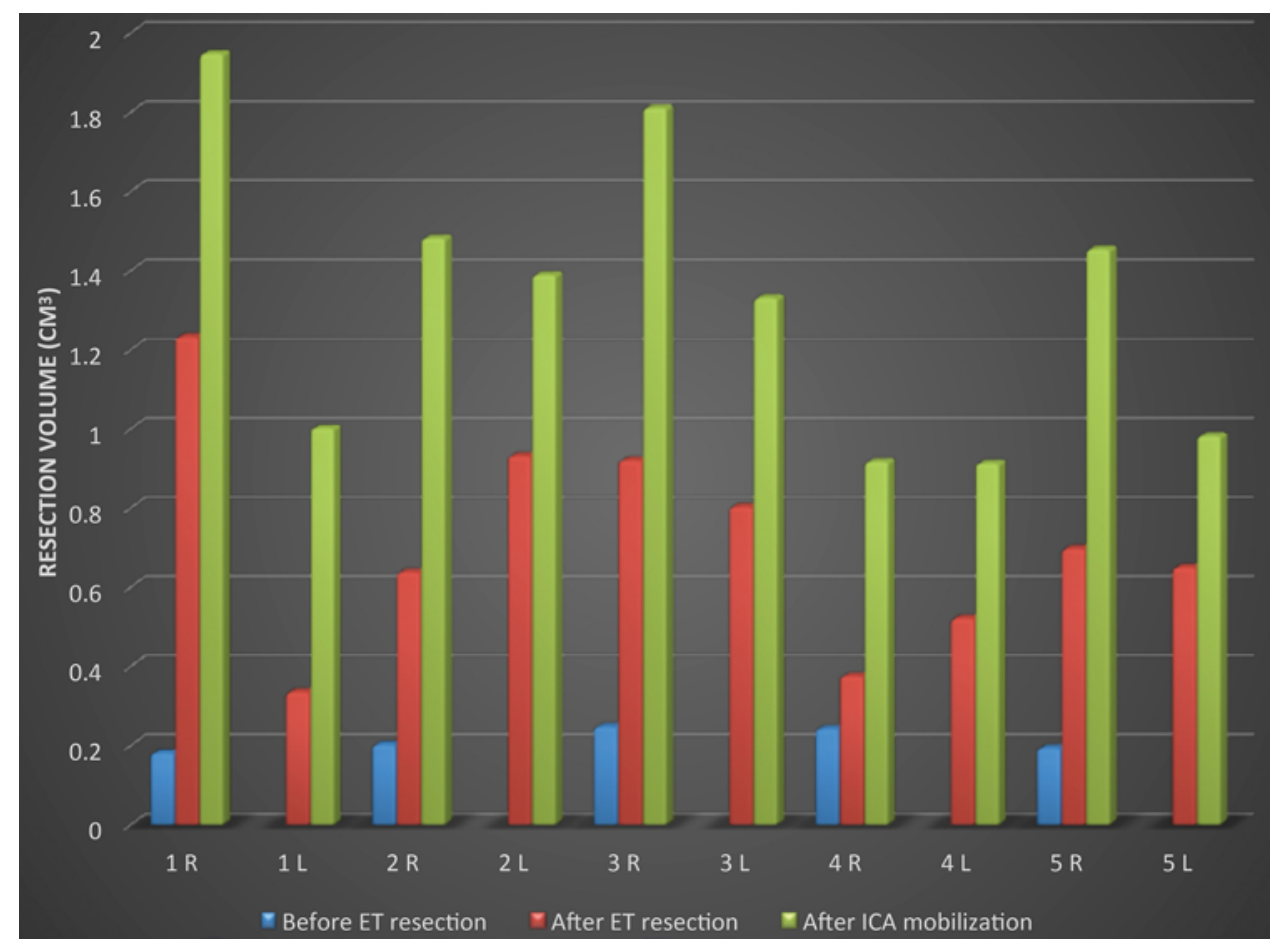

FIG. 6. 3D bar graph demonstrating resection volumes at each stage of the resection of 5 cadavers with each side denoted by $R$ (right) or L (left). Prior to ET resection, the right side was drilled in all 5 cadavers with volumes shown in blue. After ET (red) and after ICA mobilization (green), both the right and left sides were drilled in all 5 cadavers ( 10 sides). 

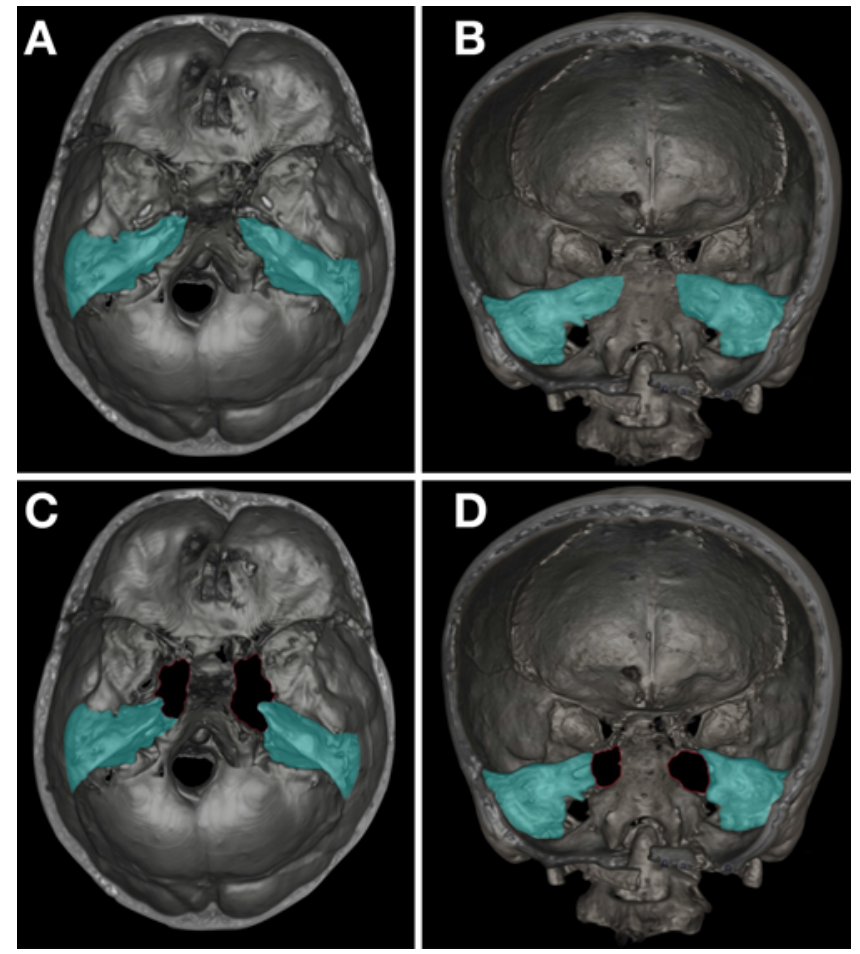

FIG. 7. Prior to dissection/petrous apex drilling, an initial fine-cut CT scan of one of the cadaver heads was imported into the Sectra Table (Sectra $A B$ ) to produce $3 D$ axial $(A)$ and coronal $(B)$ reconstructions of the petrous temporal bone (light blue). After the TMTP approach, ET resection, ICA mobilization, and petrous apex drilling, the same cadaveric head was again scanned into the Sectra Table to produce 3D reconstructions of the drilled petrous temporal bone in the axial $(\mathbf{C})$ and coronal planes (D). The empty space created by bilateral drilling of the petrous apex is demonstrated in black with red outline.

posterosuperior angulation of the drill extends access to the superior portion of the petrous apex. After completion of these steps, the ICA can be laterally mobilized approximately $4.5 \mathrm{~mm}$ to maximally expose the petrous apex. ${ }^{7}$

The reaches of the endonasal endoscopic TMTP approach with and without ET resection and/or carotid mobilization were studied, as listed in Table 3. Moreover, there have been a number of cadaveric comparative analyses examining the differences between the TMTP approach and traditional transcranial alternatives., ${ }^{2,19,28}$ The culmination of this work has been the transition from the laboratory to the operating room, where endoscopic endonasal carotid mobilization has been used in live humans without injury to the ICA..$^{29}$

In 2009, Zanation et al. ${ }^{29}$ published a series of 20 patients who underwent infrapetrous transpterygoid approaches for resection of various petrous apex pathologies, including inflammatory cystic lesions and solid tumors. The medial transsphenoidal approach without ICA mobilization was used for cystic lesions protruding into the sphenoid sinus. For other lesions for which access was hindered by the paraclival ICA, carotid mobilization was performed. Details on the carotid mobilization technique were not included. Regarding ET removal, the authors performed a $1-\mathrm{cm}$ resection of the cartilaginous ET to im-
TABLE 3. Skull base targets utilizing the endoscopic endonasal TMTP approach

\begin{tabular}{ll}
\hline \multicolumn{1}{c}{ Authors \& Year } & \multicolumn{1}{c}{ Target } \\
\hline Cavallo et al., 20054 & Pterygopalatine fossa \\
\hline Kassam et al., 2005 & $\begin{array}{c}\text { Middle 3rd of clivus, petrous apex, } \\
\text { middle fossa, infratemporal fossa }\end{array}$ \\
\hline Zanation et al., 2009 & Petrous apex \\
\hline Boari et al., 2010 & Paraclival region \\
\hline Hofstetter et al., 2010 & $\begin{array}{l}\text { Pterygopalatine fossa, infratemporal } \\
\text { fossa, petrous apex, Meckel's cave }\end{array}$ \\
\hline Taniguchi et al., 2010 & Parapharyngeal space \\
\hline Komatsu et al., 2012 & Trigeminal nerve \& Meckel's cave \\
\hline Lee et al., 2012 & Jugular foramen \\
\hline Kantola et al., 2013 & Foramen ovale \\
\hline Battaglia et al., 2014 & Infratemporal fossa \& parapharyngeal \\
& space \\
\hline Kasemsiri et al., 2013 & Petrous apex, Meckel's cave, cavern- \\
& ous sinus, infratemporal fossa \\
\hline Van Gompel et al., 2014 & Petrous apex \\
\hline Gu et al., 2015 & Meckel's cave \\
\hline Jacquesson et al., 201510,11 & Petroclival region \\
\hline Komune et al., 2015 & Jugular foramen \\
\hline Moussazadeh et al., 2015 & Petrous temporal bone \\
\hline
\end{tabular}

prove access. Boari et al. ${ }^{2}$ compared the standard Le Fort I maxillotomy to the TMTP to evaluate differences in exposure of the clival/paraclival area. Exposure afforded by the TMTP was approximately twice that of the Le Fort I maxillotomy due to the limitations imposed by the pterygoid plates in the latter approach. Petrolingual ligament division and sacrifice of the cartilaginous ET for improved carotid mobilization were performed. Morphometric comparative analysis before and after carotid mobilization was not provided. In a previous study, ${ }^{28}$ we compared petrous apex resection volumes achieved with the transcranial open anterior petrosectomy ${ }^{16}$ to those of the endoscopic anterior petrosectomy via the TMTP approach. We found that the open anterior petrosectomy results in greater resection volumes that are mostly restricted to the superior portion of the petrous apex, whereas the endoscopic anterior petrosectomy provides access to the inferior portion of the petrous apex and results in smaller resection volumes. Dynamic carotid mobilization was used during endoscopic petrous drilling; however, pre- and postmobilization resection volumes were not assessed. In this study, we supplement that previous work with additional volumetric calculations pre- and post-ET resection and carotid mobilization using static carotid retraction.

\section{Cartilaginous ET Resection}

The relationship of the ET and ICA in lateral endoscopic skull base surgery was nicely demonstrated in an anatomical study by Liu et al. in 2014. ${ }^{21}$ The authors divided the cartilaginous ET into 4 anatomical segments from anterior to posterior beginning at the torus tubarius: nasopharyngeal, pterygoid, lacerum, and petrosal. The 
cartilaginous ET limits petrous apex access and resection in the vertical axis, given its relative location to the anterior genu and horizontal petrous segment of the ICA in the horizontal or anteroposterior plane. After removal of the base of the medial and lateral pterygoid, as one proceeds from anterior to posterior along the superior surface of the nasopharyngeal, pterygoid, and anterior lacerum segments of the cartilaginous ET, ${ }^{21}$ the anterior genu of the ICA is encountered in the same plane. Therefore, the working instrument cannot easily access any petrous bone behind and/or beneath this level. Moreover, the location of the cartilaginous ET prevents lowering of the handle of the drill thereby preventing posterosuperior access behind the ICA. It has been demonstrated that removal of a portion of the cartilaginous ET improves infrapetrous access to the lower petrous apex for lesions, such as chordomas, chondrosarcomas, and cholesterol granulomas., ${ }^{228}$ More recently, the ET-sparing translacerum approach to the inferior petrous apex has been described by Taniguchi and colleagues, ${ }^{26}$ however, this approach does not afford access to the superior petrous apex. With a focus on maximal petrous apex exposure and resection in the present study, the first 3 cartilaginous ET segments were resected sparing the torus tubarius. With this maneuver, space is created to allow for subtle but necessary vertical angulation of the drill to access the inferior and midportions of the petrous apex through the infrapetrous window (Fig. 3B and C). In our cadaver model, posterior cartilaginous ET resection increased petrous apex resection volume by almost 4 times (from 0.203 to $0.709 \mathrm{~cm}^{3}$ ). However, consideration has to be given to the consequences of resecting the ET, such as middle ear pressure and pain, hearing loss, and otitis media. ${ }^{25}$ Recently, ET mobilization in the inferior direction has been described in the TMTP approach during live surgery to avoid such complications. ${ }^{22}$ In our cadaveric study, this was not found to be a feasible maneuver given the size of the cartilaginous ET and lack of space to mobilize it downward.

\section{Carotid Mobilization}

Our cadaveric results demonstrate that the entire vertical axis of the petrous apex up to the face of the middle fossa can be exposed and drilled if carotid mobilization is maximized. Subsequently, the expanded window will provide more lateral access to the petroclival region, which may result in the ability to resect challenging lesions, such as small-to-moderate sized petroclival lesions. We hypothesize that the reason for the improved petrous apex exposure and carotid mobilization is directly related to the following maneuvers that have collectively been described in previously cited papers and include 1) increased bony removal over the anterior bend of the cavernous ICA, 2) disconnection of the petrolingual ligament, 3) transection of all of the fibrocartilaginous attachments of the lacerum segment of the ICA, 4) transection of the pharyngobasilar fascia from the anterior genu, and 5) use of angled endoscopes. We have previously shown that the combination of these maneuvers allows for an average carotid mobilization of $4.5 \mathrm{~mm}$ in the axial plane and exposes more of the superior portion of the petrous apex. ${ }^{7}$ With static ICA mobilization, we were able to perform 4-handed, binostril surgery to maximize petrous apex resection. Whereas removal of the ET accesses the lower and middle portions of the petrous apex and petroclival region (Fig. 2C), paraclival carotid mobilization opens the suprapetrous window (Fig. 2D). In this cadaver study, we found that carotid mobilization after ET resection doubled volumes of petrous apex resection (from 0.709 to $1.322 \mathrm{~cm}^{3}$ ).

Although the right-sided drilling window was not statistically different from the left, there was a notable difference $\left(1.5 \mathrm{~cm}^{3}\right.$ vs $\left.1.1 \mathrm{~cm}^{3}\right)$, which we attribute to the surgeon's handedness (Table 2). For right-handed surgeons, introducing the drill from the left nostril allows more angulation/maneuverability to the contralateral retrocarotid petrous bone. This may lead to more bone resection superolaterally on the petrous bone and expands the window to the petroclival region.

\section{Approach Selection}

While the endoscopic endonasal TMTP approach with carotid mobilization avoids some of the risks associated with transcranial approaches, ${ }^{29}$ it may be associated with the following morbidities: 1) dry, uncomfortable eye from division of the vidian nerve; 2) nasal mucosal necrosis; 3) oroantral fistulas; 4) recurrent sinusitis or middle ear infections; 5) dacrocystitis; 6) injury of the nerves and arteries of the teeth leading to alveolar necrosis, hypesthesia, and/or devitalized teeth; 7) dural injury and CSF leakage; 8) cranial nerve injury resulting in facial numbness or diplopia; or 9) cerebrovascular stroke secondary to carotid injury. ${ }^{6,9}$ ET resection and subsequent dysfunction results in secretory otitis media and conductive hearing loss; however, this can be reversed with insertion of a tympanostomy tube. ${ }^{24}$ As a general rule, an optimal surgical approach should provide the most direct maximal access, not across neurovascular structures, and preferably unilateral rather than bilateral. The selection of an open versus endonasal approach to the petroclival region is governed by several factors, such as location (extra- or intradural), size, and consistency of the pathology in addition to individual anatomy. For soft, extradural expansive lesions, such as chordomas/chondrosarcomas, Meckel's cave, or petrous apex lesions, the surgical corridor is predefined, and endonasal access is facilitated by the tumor. In such cases, neurovascular structures are mostly mobilized by the tumor, and the risk of injury is less in expert hands. Firm, intradural lesions such as petroclival meningiomas are more challenging, due to preserved bony anatomy of the lateral skull base. Recently, Jacquesson et al. published 2 studies $^{10,11}$ that examined the differences between the open anterior petrosectomy and endoscopic approaches to the petroclival region. They concluded that the transcranial anterior petrosectomy should be used for the removal of medium-sized petrous apex intradural tumors, whereas expanded endoscopic approaches should be reserved for extradural midline tumors. The question that we attempted to answer in our study is the size limitation. The creation and expansion of a surgical access window in the petroclival region requires challenging, morbid maneuvers (ET resection and ICA mobilization), and the maximum window achieved was $1.5 \mathrm{~cm}^{3}$, as shown by our volumetric analysis. In light of the results of this study, we propose 
that firm, intradural lesions larger than $1.5 \mathrm{~cm}^{3}$ located lateral to the petrosphenoid suture should be considered for an open transcranial approach. The medial expansion of the petroclival window by further drilling of the clivus is more suited for more medial lesions. Also, a contralateral transpterygoid approach might achieve a larger window to the petroclival region.

\section{Limitations of the Study}

The cadaveric nature of this study should be factored into the interpretation of any volumetric results due to the limited number of specimens studied, altered normal tissue elasticity, and absence of pathology. These results must be further validated in live humans. However, we consider this a step in the right direction of defining the role of the relatively new endoscopic approaches the skull base.

\section{Conclusions}

The transpterygoid corridor to the petroclival region is maximally expanded by the resection of the cartilaginous ET and mobilization of the paraclival ICA. These added maneuvers expanded the deep window 6 times and provided more lateral access to the petroclival region with a maximum volume of $1.5 \mathrm{~cm}^{3}$. This may result in the ability to resect small to moderate size intradural petroclival lesions up to that volume. Larger lesions may better be approached through an open transcranial approach.

\section{References}

1. Battaglia P, Turri-Zanoni M, Dallan I, Gallo S, Sica E, Padoan G, et al: Endoscopic endonasal transpterygoid transmaxillary approach to the infratemporal and upper parapharyngeal tumors. Otolaryngol Head Neck Surg 150:696-702, 2014

2. Boari N, Roberti F, Biglioli F, Caputy AJ, Mortini P: Quantification of clival and paraclival exposure in the Le Fort I transmaxillary transpterygoid approach: a microanatomical study. J Neurosurg 113:1011-1018, 2010

3. Cavallo LM, Messina A, Cappabianca P, Esposito F, de Divitiis E, Gardner P, et al: Endoscopic endonasal surgery of the midline skull base: anatomical study and clinical considerations. Neurosurg Focus 19(1):E2, 2005

4. Cavallo LM, Messina A, Gardner P, Esposito F, Kassam AB, Cappabianca P, et al: Extended endoscopic endonasal approach to the pterygopalatine fossa: anatomical study and clinical considerations. Neurosurg Focus 19(1):E5

5. Chatrath P, Nouraei SA, De Cordova J, Patel M, Saleh HA: Endonasal endoscopic approach to the petrous apex: an image-guided quantitative anatomical study. Clin Otolaryngol 32:255-260, 2007

6. DeFreitas J, Lucente FE: The Caldwell-Luc procedure: institutional review of 670 cases: 1975-1985. Laryngoscope 98:1297-1300, 1988

7. Freeman JL, Sampath R, Casey MA, Quattlebaum SC, Ramakrishnan VR, Youssef AS: Transposition of the paraclival carotid artery: a novel concept of self-retaining vascular retraction during endoscopic endonasal skull base surgery technical report. Acta Neurochir (Wien) 158:1625-1629, 2016

8. Gu Y, Yu Y, Zhang X, Hu F, Wang X, Xu W, et al: Endoscopic endonasal transmaxillary transpterygoid approach to Meckel cave: anatomical study and preliminary clinical results. J Neurol Surg A Cent Eur Neurosurg 76:205-210, 2015
9. Hofstetter CP, Singh A, Anand VK, Kacker A, Schwartz TH: The endoscopic, endonasal, transmaxillary transpterygoid approach to the pterygopalatine fossa, infratemporal fossa, petrous apex, and the Meckel cave. J Neurosurg 113:967-974, 2010

10. Jacquesson T, Berhouma M, Tringali S, Simon E, Jouanneau $\mathrm{E}$ : Which routes for petroclival tumors? A comparison between the anterior expanded endoscopic endonasal approach and lateral or posterior routes. World Neurosurg 83:929936, 2015

11. Jacquesson T, Simon E, Berhouma M, Jouanneau E: Anatomic comparison of anterior petrosectomy versus the expanded endoscopic endonasal approach: interest in petroclival tumors surgery. Surg Radiol Anat 37:1199-1207, 2015

12. Kantola VE, McGarry GW, Rea PM: Endonasal, transmaxillary, transpterygoid approach to the foramen ovale: radioanatomical study of surgical feasibility. J Laryngol Otol 127:1093-1102, 2013

13. Kasemsiri P, Solares CA, Carrau RL, Prosser JD, Prevedello DM, Otto BA, et al: Endoscopic endonasal transpterygoid approaches: anatomical landmarks for planning the surgical corridor. Laryngoscope 123:811-815, 2013

14. Kassam AB, Gardner P, Snyderman C, Mintz A, Carrau R: Expanded endonasal approach: fully endoscopic, completely transnasal approach to the middle third of the clivus, petrous bone, middle cranial fossa, and infratemporal fossa. Neurosurg Focus 19(1):E6, 2005

15. Kassam AB, Vescan AD, Carrau RL, Prevedello DM, Gardner P, Mintz AH, et al: Expanded endonasal approach: vidian canal as a landmark to the petrous internal carotid artery. $\mathbf{J}$ Neurosurg 108:177-183, 2008

16. Kawase T, Toya S, Shiobara R, Mine T: Transpetrosal approach for aneurysms of the lower basilar artery. J Neurosurg 63:857-861, 1985

17. Klossek JM, Ferrie JC, Goujon JM, Fontanel JP: Endoscopic approach of the pterygopalatine fossa: report of one case. Rhinology 32:208-210, 1994

18. Komatsu F, Komatsu M, Di Ieva A, Tschabitscher M: Endoscopic approaches to the trigeminal nerve and clinical consideration for trigeminal schwannomas: a cadaveric study. $\mathbf{J}$ Neurosurg 117:690-696, 2012

19. Komune N, Komune S, Matsushima K, Rhoton AL Jr: Comparison of lateral microsurgical preauricular and anterior endoscopic approaches to the jugular foramen. J Laryngol Otol 129 (Suppl 2):S12-S20, 2015

20. Lee DL, McCoul ED, Anand VK, Schwartz TH: Endoscopic endonasal access to the jugular foramen: defining the surgical approach. J Neurol Surg B Skull Base 73:342-351, 2012

21. Liu J, Pinheiro-Neto CD, Fernandez-Miranda JC, Snyderman $\mathrm{CH}$, Gardner PA, Hirsch BE, et al: Eustachian tube and internal carotid artery in skull base surgery: an anatomical study. Laryngoscope 124:2655-2664, 2014

22. Maurer AJ, Bonney PA, Iser CR, Ali R, Sanclement JA, Sughrue ME: Endoscopic endonasal infrapetrous transpterygoid approach to the petroclival junction for petrous apex chondrosarcoma: technical report. J Neurol Surg Rep 76:e113-e116, 2015

23. Moussazadeh N, Kulwin C, Anand VK, Ting JY, Gamss C, Iorgulescu JB, et al: Endoscopic endonasal resection of skull base chondrosarcomas: technique and early results. J Neurosurg 122:735-742, 2015

24. Neel HB III, Keating LW, McDonald TJ: Ventilation in secretory otitis media: effects on middle ear volume and eustachian tube function. Arch Otolaryngol 103:228-231, 1977

25. Schilder AGM, Bhutta MF, Butler CC, Holy C, Levine LH, Kvaerner KJ, et al: Eustachian tube dysfunction: consensus statement on definition, types, clinical presentation and diagnosis. Clin Otolaryngol 40:407-411, 2015

26. Taniguchi M, Akutsu N, Mizukawa K, Kohta M, Kimura H, 
Kohmura E: Endoscopic endonasal translacerum approach to the inferior petrous apex. J Neurosurg 124:1032-1038, 2016

27. Taniguchi M, Kohmura E: Endoscopic transnasal transmaxillary transpterygoid approach to the parapharyngeal space: an anatomic study. Minim Invasive Neurosurg 53:255-260, 2010

28. Van Gompel JJ, Alikhani P, Tabor MH, van Loveren HR, Agazzi S, Froelich S, et al: Anterior inferior petrosectomy: defining the role of endonasal endoscopic techniques for petrous apex approaches. J Neurosurg 120:1321-1325, 2014

29. Zanation AM, Snyderman CH, Carrau RL, Gardner PA, Prevedello DM, Kassam AB: Endoscopic endonasal surgery for petrous apex lesions. Laryngoscope 119:19-25, 2009

30. Ziyal IM, Salas E, Wright DC, Sekhar LN: The petrolingual ligament: the anatomy and surgical exposure of the posterolateral landmark of the cavernous sinus. Acta Neurochir (Wien) 140:201-205, 1998

\section{Disclosures}

The authors report no conflict of interest concerning the materi- als or methods used in this study or the findings specified in this paper.

\section{Author Contributions}

Conception and design: Youssef, Freeman, Sampath, Quattlebaum, Ramakrishnan. Acquisition of data: Youssef, Freeman, Sampath, Quattlebaum, Casey. Analysis and interpretation of data: Youssef, Freeman, Sampath, Quattlebaum. Drafting the article: Youssef, Freeman, Sampath, Ramakrishnan. Critically revising the article: all authors. Reviewed submitted version of manuscript: all authors. Approved the final version of the manuscript on behalf of all authors: Youssef. Statistical analysis: Youssef, Freeman, Quattlebaum, Ramakrishnan. Administrative/technical/ material support: Youssef, Freeman. Study supervision: Youssef, Freeman. Figure editing: Folzenlogen, Freeman.

\section{Correspondence}

A. Samy Youssef, Department of Neurosurgery, University of Colorado, 12631 E 17th Ave., C307, Aurora, CO 80045. email: samy.youssef@ucdenver.edu. 